\title{
Dwarf galaxy candidates in the NGC 3665 galaxy group
}

\author{
Bettina Ogbuagu-Poledna \\ and Werner W. Zeilinger \\ Institute of Astronomy, Türkenschanzstr. 17, A-1180 Vienna, Austria \\ email: poledna@astro.univie.ac.at
}

\begin{abstract}
We present preliminary results of a search for candidate dwarf galaxies in the NGC 3665 galaxy group. A $0.1 \times 0.1 \mathrm{Mpc}^{2}$ field around the dominant early-type galaxy NGC 3665 was investigated using BRI images from the $2.5 \mathrm{~m}$ INT WFC archive. A search with the SExtractor yielded 148 dwarf galaxy candidates in the range $-12>M_{B}>-16$. The resulting luminosity function for the group can be described by a standard Schechter function.
\end{abstract}

Keywords. galaxies: dwarf, galaxies: evolution

\section{Introduction}

Present-day galaxy groups contain a significant fraction of the galaxy population of the local universe. It is therefore of crucial importance to understand the dynamical and chemical galaxy evolution in such environments. Galaxy groups are an ideal laboratory to study not only galaxy-galaxy interactions but also the evolution of cosmic structures at a "cellular" level. The detection of a diffuse hot intra-group medium in a number of groups ascertained their physical reality; e.g. Mulchaey et al. (1996), Ponman et al. (1996). Dwarf galaxies are an important indicator for the dynamical evolution of a group. Zabludoff \& Mulchaey (1998) detected a significant dwarf galaxy population down to $M_{B} \simeq-14$ in X-ray bright groups which is apparently absent in X-ray non-detected groups of the same optical morphology. The importance of galaxy-galaxy interactions in group environments is illustrated by the fact that bulge-dominated systems are more common and that disk systems with apparent morphological signatures of interactions show higher star formation rates than in the field, e.g. Tran et al. (2001).

\section{Characteristic Properties of the NGC 3665 Group}

The NGC 3665 galaxy group was first mentioned by Geller \& Huchra (1983) and subsequently described by Tully (1988) and Garcia (1993). The group is located at a distance of $27.6 \mathrm{Mpc}\left(\mathrm{H}_{0}=75 \mathrm{~km} \mathrm{~s}^{-1} \mathrm{Mpc}^{-1}\right)$ and appears as a loose system of mostly late-type galaxies around the dominant early-type galaxy NGC 3665. A search of an area of $1 \mathrm{Mpc}^{2}$ around NGC 3665 resulted in only 10 luminous group members yielding a velocity dispersion of $65 \mathrm{~km} \mathrm{~s}^{-1}$ for the group. However, velocity dispersions of at least $100-200 \mathrm{~km} / \mathrm{s}$ are expected for a virialized group by relating the minimum density of a group to the density of the universe (Mamon 1994). Therefore the derived velocity dispersion of the NGC 3665 group remains suspect. The presence of a hot diffuse intra-group medium in the group has been confirmed and it belongs to the faintest and coolest systems (Helsdon et al. 2005). BRI images obtained from the WFC archive of the $2.5 \mathrm{~m}$ INT were analyzed in order to search for further group member candidates. The images were centered upon NGC 3665 yielding a field of view of $\approx 34^{\prime} \times 34^{\prime}$ which corresponds to about $0.1 \mathrm{Mpc}^{2}$. 


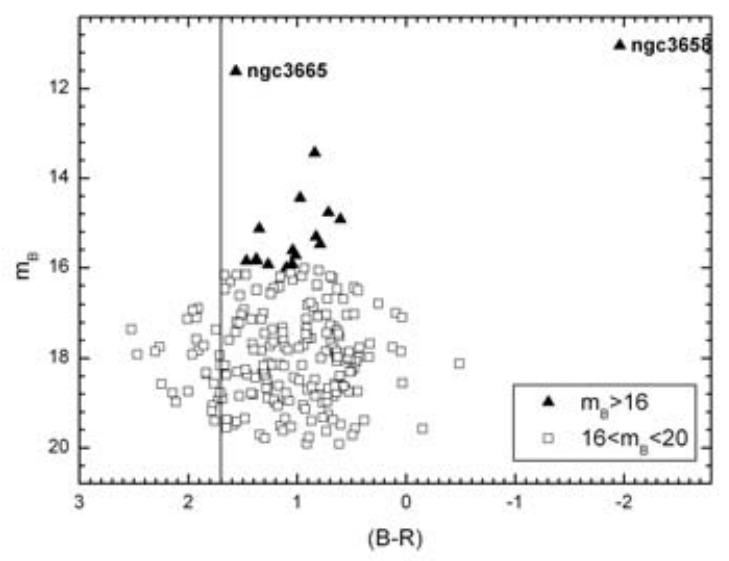

Figure 1. Colour-magnitude diagram of all group member candidates.

Standard procedures were applied to calibrate the images photometrically. The candidate search was performed using the SExtractor (Bertin \& Arnouts (1996)). The search based upon a stellaricity indix of $<0.9, m_{B}<16$ for bright galaxies and $16<m_{B}<20$ for dwarf galaxies. As a minimum size for dwarf galaxies a value of $1.5^{\prime \prime}$ was adopted based upon the seeing measurements.

\section{Preliminary Results}

A (B-R)-diagramm for all likely members was constructed (see Figure 1). After applying a cut-off at $(\mathrm{B}-\mathrm{R})=1.7$ (as it is applied in Miles et al. (2004)), 17 bright and 148 dwarf candidate galaxies remain. The ensure the group membership, spectroscopic observations will be carried out. 26 galaxies of all candidates were previously known, 139 are new objects. Only two (NGC 3665 and NGC 3658) have known redshifts. The luminosity function of the group members follows the shape of the Schechter function with a dip around $M_{\mathrm{B}}=-18$ which is in good agreement with luminosity functions of groups presented in recent publications, e.g. Miles et al. (2004).

\section{Acknowledgements}

The research project is supported by the Austrian Science Fund (FWF project P14783).

\section{References}

Bertin, E. \& Arnouts, S. 1996, A\&AS 117, 393

Garcia, A.M. 1993, A\&AS 100, 47

Geller, M.J. \& Huchra, J.P. 1983, ApJS 52, 61

Helsdon, S.F., Ponman, T.J. \& Mulchaey, J.S. 2005, ApJ 618, 679

Mamon, G.A. 1994, in: F. Durret et al. (eds.) Clusters of Galaxies, Gif-sur-Yvette, Edition Frontières, p. 291

Miles, T.A., Raychaudhury, S., Forbes, D.A., Goudfrooij, P., Ponman, T.J. \& Kozhurina-Platais, V. 2004, MNRAS 355, 785

Mulchaey, J.S., Davis, D.S., Mushotzky, R.F. \& Burstein, D. 1996, ApJ 456, 80

Ponman, T.J., Bourner, P.D.J., Ebeling, H. \& Bohringer, H. 1996, MNRAS 283, 690

Tran, K-V.H., Simard, L., Zabludoff, A.I. \& Mulchaey, J.S. 2001, ApJ 549, 172

Tully, R.B. \& Fisher, J.R. 1988 Catalog of Nearby Galaxies, Cambridge University Press

Zabludoff, A.I. \& Mulchaey, J.S. 1998, ApJ 496, 73 